\title{
Relative validation of the dietary intake of fatty acids among adults in the Swedish National Dietary Survey using plasma phospholipid fatty acid composition
}

\author{
Eva Warensjö Lemming ${ }^{1}{ }^{*}$, Cecilia Nälsén ${ }^{1}$, Wulf Becker ${ }^{1}$, Peter Ridefelt ${ }^{2}$, Iréne Mattisson ${ }^{1}$ and \\ Anna Karin Lindroos ${ }^{1}$ \\ ${ }^{1}$ National Food Agency, Uppsala, Sweden \\ ${ }^{2}$ Department of Medical Sciences, Clinical Chemistry, Uppsala University, Uppsala, Sweden
}

(Received 19 June 2014 - Final revision received 1 December 2014 - Accepted 2 December 2014)

Journal of Nutritional Science (2015), vol. 4, e25, page 1 of 9

doi:10.1017/jns.2015.1

Abstract

The aims of a national dietary study are several-fold. One purpose is to monitor the intake of foods and nutrients in the population and to compare intakes with dietary recommendations. It is, however, difficult to measure dietary fat intake and plasma biomarker fatty acid (FA) composition may be used as an objective measure of dietary fat intake. Thus, the relative ability of a diet record to capture habitual fat intake was validated against biomarker FA. Dietary fat intake was measured in a novel self-assisted web-based 4-d food record - the 'Riksmaten' method. Spearman rank correlations between dietary FA, certain food groups (fish-shellfish, dairy products, meat and sausages, and spreads) and the fat content of these food groups and biomarker FA were explored. Participants were 150 women and 129 men, aged 18-80 years, who took part in the Swedish National Dietary Survey, Riksmaten adults 2010-11. Blood samples were collected on average $20 \mathrm{~d}$ after the diet record and FA composition was measured in plasma phospholipids by GLC. Total $n$-3 FA $(r$ 0.31), EPA $(r 0.34)$ and DHA $(r$ 0.42) were correlated between plasma and diet (all $P \leq 0 \cdot 001)$. Adjustment for covariates attenuated the relationships. Linoleic acid was only marginally correlated $(r 0.15 ; P=0.06)$ in women. Plasma pentadecaenoic acid and heptadecaenoic acid were correlated with dairy product intake as previously reported. In conclusion, the Riksmaten method appears valid for the purpose of collecting data on dietary fat composition, at least in a healthy adult population.

Key words: Fatty acid intake: National dietary studies: Swedish National Dietary Survey: Phospholipids: Plasma biomarkers

There is a continuous interest in dietary fats and their association with chronic disease and especially $\mathrm{CVD}^{(1)}$. Dietary fatty acids are biologically active compounds that participate in various pathways and regulate the fluidity of biological membranes. Further, PUFA regulate gene expression and are precursors of eicosanoids ${ }^{(2)}$. The current dietary recommendations advise us to decrease the intake SFA and simultaneously to increase the intake of unsaturated fatty acids ${ }^{(3)}$ to reduce the risk of $\mathrm{CVD}^{(4)}$ and is a cornerstone in public health nutrition. One main dietary advice in Sweden ${ }^{(5)}$ is to consume fish 2-3 times per week (of which one portion should be fatty fish) since fish consumption is associated with established cardioprotective benefits ${ }^{(6)}$ and contributes essential nutrients, such as $n$ - 3 fatty acids (EPA and DHA) and vitamin D. It is recommended that $n$-3 fatty acids account for a minimum of $1 \%$ of energy intake ${ }^{(3)}$.

In order to monitor and evaluate the intake of, for example, fish and $n$-3 fatty acids in the population the results from the Swedish National Dietary Survey is used. In the latest survey, Riksmaten adults 2010-11 ${ }^{(7)}$, a newly developed web-based self-assisted $4 \mathrm{~d}$ dietary record was used to collect diet data. A diet record or a $24 \mathrm{~h}$ recall is considered to give more

Abbreviations:: LA, linoleic acid; NFA, National Food Agency; PL, phospholipid.

* Corresponding author: Dr Eva Warensjö Lemming, email Eva.WarensjoLemming@slv.se 
accurate dietary intake data than, for example, $\mathrm{FFQ}^{(8,9)}$. However, in any dietary survey, including our national dietary survey, there is an inherent difficulty in measuring dietary exposure and especially dietary fat ${ }^{(10,11)}$. This is connected to random and systematic measurement errors, the difficulty in estimating portion sizes as well as inadequacies in food composition databases, for example the use of standard fat in recipes. Under-reporting is common in all dietary survey techniques and may lead to spurious diet-disease relationships ${ }^{(12)}$. Moreover, the degree of under-reporting is affected by different factors, for example the BMI among participants, and this is particularly true for foods perceived as unhealthy ${ }^{(10-13)}$.

Biomarker fatty acids may be used as indicators of dietary fat. However, fatty acid biomarkers do not provide an accurate reflection of absolute intake, partly because fatty acid composition is influenced by endogenous metabolism and remodelling ${ }^{(14,15)}$ as well as to sex, genotype, fat intake level, BMI, smoking status and exercise ${ }^{(16)}$. Certain fatty acids such as linoleic acid (LA; $18: 2 n-6$ ), EPA and DHA provide a better reflection of the dietary content, while others such as SFA and MUFA provide a worse estimate of dietary intake because of endogenous metabolism. The biomarker fatty acid composition in the phospholipid (PL) and cholesteryl ester fraction of lipoprotein particles reflects the dietary fat quality during the last days and weeks, respectively, while fatty acids of other fractions such as erythrocyte membranes and adipose tissue reflect the intake of those fatty acids during the last months and year(s), respectively ${ }^{(15,17-21)}$. Thus, the relative validity of the intake of dietary fatty acids from a dietary survey may be validated using biomarker fatty acids.

The novel web-based Riksmaten method, a 4-d web-based dietary record used in the latest Swedish National Dietary Survey, Riksmaten adults $2010-11$, is currently being validated. The present study aimed to investigate the relative validity of the estimated dietary intake of fat, especially EPA and DHA and LA, using biomarker fatty acids measured in plasma PL in a subsample of Riksmaten adults 2010-11. We also investigated associations between intake of certain food groups and amount of fat from the food groups - fish and shellfish, spreads (margarines, dairy-based spreads and butter), meat and sausages and dairy products (milk, yoghurt, cream, sour cream and cheese) - and corresponding biomarker fatty acids in plasma PL. In addition, the ability of the questionnaire to capture intake of $n$-3 fatty acids from fish-shellfish intake was explored.

\section{Experimental methods}

\section{Study design and population}

Riksmaten adults 2010-11 is the latest Swedish National Dietary Survey conducted by the National Food Agency (NFA) in association with Statistics Sweden. The study was carried out between May 2010 and July 2011 to capture seasonal variations. A nationwide random sample $(n$ 5003) was selected and $36 \%$ (1005 women and 792 men) partook in the dietary survey ${ }^{(7)}$. The invited sample was also requested to complete a questionnaire. In a subsample ( $n$ 1008) blood and spot urine samples were collected to measure markers of nutritional status and exposure to toxic contaminants (see Fig. 1). All participants in the present study completed the diet record, the questionnaire and donated blood samples on average $20 \mathrm{~d}$ after the dietary registration was initiated.

Sweden has seven Occupational and Environmental Medicine Centre regions. From each of these seven regions, the regional capital (Linköping, Lund, Stockholm, Umeå, Uppsala, Gothenburg, Örebro) and two randomly chosen municipalities were chosen and an equal number of individuals was randomly selected in each region independent of population size. Altogether, 1008 individuals aged between 18 and 80 years old were invited to participate in the blood sampling (twelve individuals per municipality) evenly distributed over the year. The selection of study participants was performed by Statistics Sweden, and the Occupational and Environmental Medicine Centres carried out the recruitment of the participants. In total, 300 individuals provided blood samples (participation rate $30 \%$; see Fig. 1). The study was approved by the local ethics committee at Uppsala University and all participants gave oral consent to participate.

\section{Dietary assessment}

The diet record ran over four consecutive days and was entered by the participants in a web-application (self-assisted), developed by the NFA. A public version of this is available at http://www.slv.se/sv/grupp1/Mat-och-naring/kostrad/Testmatvanekollen/ (in Swedish). The starting day was randomly allocated for the participants to capture consumption on all days of the week. Participants were instructed to record everything that they ate and drank during these days. The food list in the web application contained more than 1900 food items and dishes. A portion guide, household measures, numbers of portions (cups, pieces, slices) and grams were used to estimate the amounts eaten. The portion guide included twenty-four different food categories, with four to eight different reference sizes in each category. The application (version 04.1) is linked to the national food composition database (Livsmedelsdatabasen, version Riksmaten adults 2010-11) at the NFA, which enables automatic estimation of nutrient values and energy intake. Intake of food groups (g), energy and nutrient intakes per $\mathrm{d}$ were calculated as the average intake over the four record days. Information on physical activity level at work and leisure time was collected as part of the dietary record on a four-grade scale. Under-reporting was calculated using the Goldberg cutoff according to Black ${ }^{(22)}$ in the entire Riksmaten survey population and defines the probability of adequately reported energy, based on body size and physical activity level.

\section{Questionnaire and other data}

The questionnaire was used to collect background data, other characteristics and information on foods less frequently consumed (episodic foods). Information on self-reported weight and height was also collected in the questionnaire. BMI was calculated as weight (in $\mathrm{kg}$ ) divided by height (in $\mathrm{m}$ ) squared. The consumption frequency of different types of fish and 


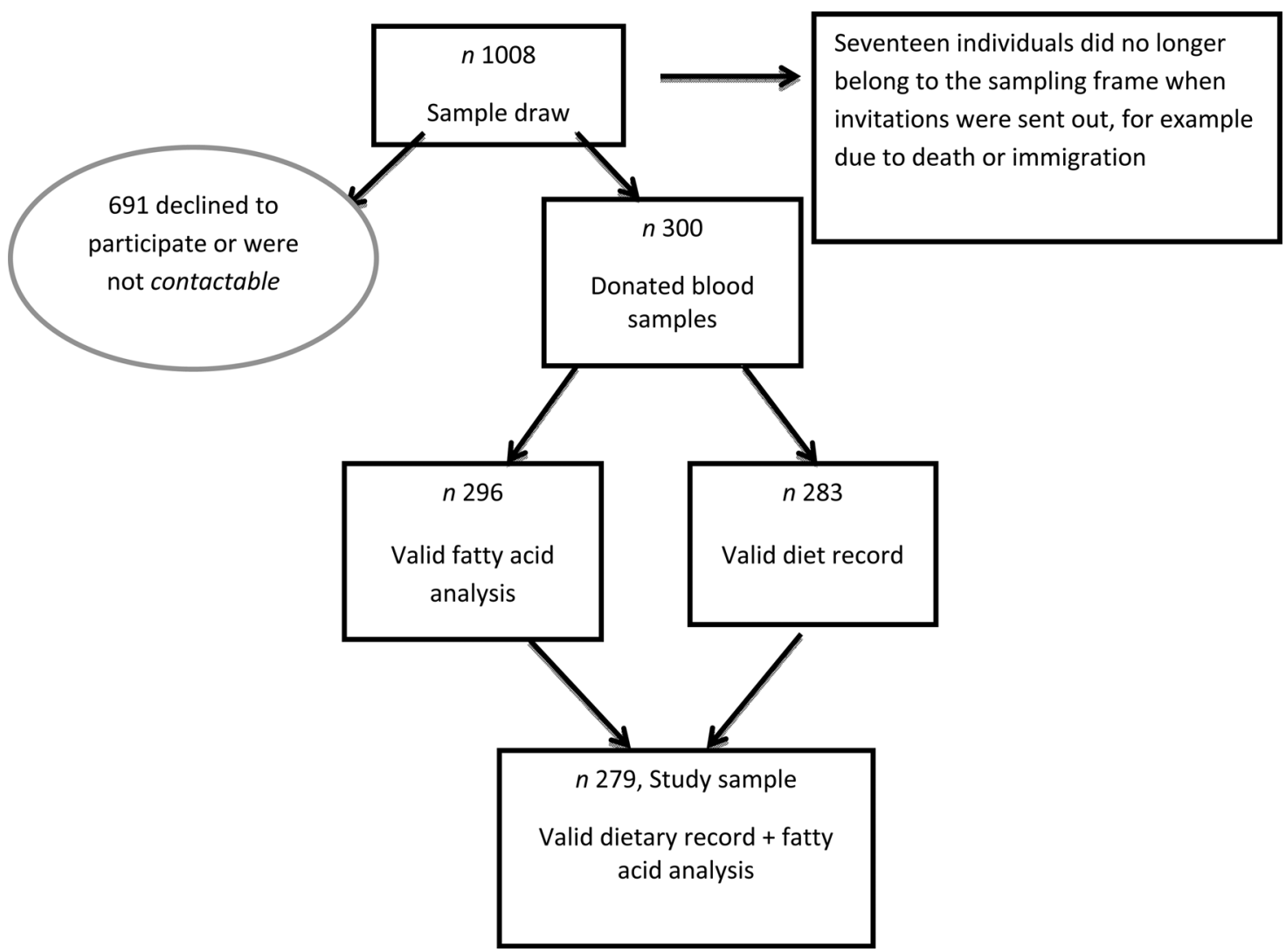

Fig. 1. Flowchart depicting the study sample. The study sample of 1008 refers to the subsample in the Riksmaten adults $2010-11$ study.

shellfish during the previous year was collected. The information from this question was used to create a three-level categorical weekly fish intake variable, with the levels $<1$ time per week, 1-2 times per week, $>2$ times per week. The consumption frequency (ranging from never to every day) ${ }^{(23)}$ of salmon, lean white fish (for example cod and saithe), herring and mackerel, and shrimp, crayfish and lobster was used to create this variable. Information on supplement use of fish oil and $n-3$ capsules reported in the questionnaire was used in sensitivity analysis. Data on age and sex of participants were collected from the Swedish population register.

\section{Sampling of blood}

Non-fasting blood was sampled at the Occupational and Environmental Medicine Centre in each region. Blood was drawn from an antecubital vein. Plasma was separated and frozen $\left(-20^{\circ} \mathrm{C}\right)$ at the regional centres. The samples were then transported frozen to the Department of Clinical Chemistry, University Hospital Uppsala and then distributed via the NFA for analyses.

\section{Analysis of plasma fatty acid composition in phospholipids}

Fatty acid composition in plasma PL was analysed at Uppsala University as previously described ${ }^{(24)}$. Briefly, methanol was added to plasma for lipid extraction. PL were separated by TLC before inter-esterification with acidic methanol at $85^{\circ} \mathrm{C}$ for $2 \mathrm{~h}$. To avoid contamination of the GLC column, non- esterified cholesterol liberated in the reaction was removed by an aluminium oxide column. The percentage composition of methylated fatty acids (14:0 to $22: 6$ ) was determined by GC with a flame ionisation detector and $\mathrm{He}$ as the carrier gas. A previous larger population study concluded that fasting state did not influence associations between fatty acids in PL and diet ${ }^{(25)}$.

\section{Statistical analysis}

The normality of the variables was checked with the ShapiroWilk test. Non-normal variables were log-transformed to improve normality. Plasma fatty acids in PL are expressed as weight percentage of total fatty acids. Food group and nutrient intakes were adjusted for total energy intake using the residual $\operatorname{method}^{(23)}$. The investigated food groups were fish and shellfish, spreads, meat and sausages, and dairy products (milk, yoghurt, fermented milk cream, sour cream and cheese). The spreads were margarines, dairy-based spread and butter used on bread or as a side dish (for example on boiled potatoes). The intake of fish-shellfish and dairy products, combined with data on fat content, was used to derive the amount of fat (in $\mathrm{g} / \mathrm{d}$ ) from the respective food group. The dietary intake of individual fatty acids (weight percentage) were treated as the percentage of total fat intake since this is conceptually similar to the way that fatty acids in plasma PL are expressed ${ }^{(25,26)}$.

The crude relationship between plasma fatty acids and dietary fatty acids, food groups and fat from food groups as well as fish-shellfish intake from the questionnaire was tested with Spearman rank correlations. Partial correlation coefficients 
were adjusted for BMI (continuous variable), smoking habits (categorical variable), physical activity level (categorical variable), alcohol intake and age (both continuous variables). In order to test for effect modification by sex we analysed the association between fatty acids in plasma and diet with linear regression analysis and introduced an interaction term between dietary fatty acid and sex.

Differences in characteristics between women and men were tested by $t$ test, $\chi^{2}$ test and the Wilcoxon rank-sum test. ANOVA was used to test for group differences and analyses were stratified on sex. The agreement between the diet record and questionnaire to rank individuals in terms of fish-shellfish intake (tertiles) was evaluated in weighted kappa $\left(\kappa_{\mathrm{w}}\right)$ statistics.

The influences of under-reporting and supplement use (fishoil and $n$ - 3 capsules) were accounted for in sensitivity analyses. $P$ values $<0.05$ was considered significant. All analyses were carried out in STATA version 12.1 (STATA Corp.).

\section{Results}

\section{Background and dietary characteristics}

Table 1 depicts characteristics of study participants. More women ( $n$ 150; $54 \%$ ) than men ( $n$ 129; $46 \%$ ) participated in the study and women were on average younger. Physical activity level, smoking habits and number of under-reporters did not differ between the sexes. There were small differences in food intake and fat intake (expressed as $\mathrm{g} / 10 \mathrm{MJ}$ ) between men and women, except that women had a higher intake of dairy products $(P=0.03)$ and men had a higher intake of spreads $(P<0 \cdot 01)$. In relation to the other participants in the Swedish National Dietary Survey ( $n$ 1518) the intakes of protein, carbohydrate, dietary fibre, alcohol and total fat (energy percentage) were not different (data not shown). However, the participants who donated blood samples reported significantly higher energy intake $(8.6$ v. $8.2 \mathrm{MJ}$; $P=0 \cdot 004)$. The relative proportions of fatty acids measured in plasma PL are depicted in Table 2. Age was positively associated with the proportions of plasma PL EPA and DHA while negatively associated with plasma LA, $\alpha$-linolenic acid and oleic acid. About $20 \%$ of the participants had not consumed fish according to the diet record.

\section{Correlations between plasma and dietary fatty acids}

The crude Spearman rank correlations between proportions of fatty acids in plasma PL and dietary fatty acids are presented in Table 3. In general, plasma $n-3$ fatty acids showed the strongest crude correlations with diet: $r 0.34$ for EPA and $r 0.42$ for DHA (all $P \leq 0 \cdot 001)$. Myristic acid (14:0) $(r 0 \cdot 16 ; P<$ $0 \cdot 01)$ as well as total PUFA $(r 0.12 ; P=0 \cdot 05)$ were correlated between PL and diet. PL LA (18: $2 n-6)$ was not significantly correlated with dietary intake $(r 0.07 ; P=0.24)$. Repeating these analyses with the dietary fatty acids expressed as $\mathrm{g} / \mathrm{d}$ or residual adjusted $\mathrm{g} / \mathrm{d}$ rendered similar results (data not shown). The relationships for myristic acid (14:0), total MUFA and PUFA and LA were only significant in one of the sexes. Further, for palmitoleic acid, total PUFA and LA the correlation coefficients went in opposite directions in men and women and this was confirmed as significant interactions with sex in the linear regression analysis: palmitoleic acid $(P$ for interaction $=0 \cdot 028)$, total PUFA $(P$ for interaction $=$ $0 \cdot 031)$ and LA $(P=0.034)$.

Adjusted correlations (partial) between plasma PL and dietary fatty acids were in most cases attenuated. Total $n-3$ fatty acids $(r 0.19 ; P<0 \cdot 01)$ as well as EPA $(r 0.18 ; P<0.01)$ and DHA $(r 0.33 ; P<0.001)$ remained correlated. The negative correlation between total MUFA in plasma and diet was stronger after adjustment $(r-0 \cdot 17 ; P=0.006)$ and for individual SFA most correlations remained unchanged. In men, the total SFA amount in plasma was inversely related to diet $(r-0 \cdot 18 ; P=0 \cdot 01)$ after adjustment.

\section{Correlations between plasma fatty acids and food groups}

Dairy products. The intake of dairy products $(\mathrm{g} / \mathrm{d})$ correlated with plasma PL $14: 0(r 0.15 ; P<0 \cdot 01)$ and $15: 0(r 0 \cdot 25 ; P<$ $0 \cdot 001)$ but not with $17: 0(r 0 \cdot 08 ; P=0 \cdot 2)$ in all participants, and differed between men and women. Adding butter (recorded as a spread by twenty-three participants) to the dairy product variable did not change the correlations (data not shown). Total fat and SFA intakes from dairy products were significantly correlated with plasma PL 15:0 and 17:0, but not with 14:0 and correlations differed between the sexes (Table 4). The partial correlation analyses showed that PL 15:0 remained and 17 : 0 became significantly correlated with the intake of dairy products in women. Further, the correlations between intake of total fat and SFA from dairy products disappeared for 15 : 0 but remained for 17 : 0 in women. In men, plasma PL 15 : 0 was correlated with the intake of dairy products as well as with the intake of total fat and SFA from dairy products (Table 4). PL 15 : 0 and $17: 0$ did not correlate with dietary fish-shellfish intake (data not shown).

Fish and shellfish. Intakes of total fish-shellfish and total fat and PUFA from fish- shellfish from the diet record were well correlated with very-long-chained $n-3$ fatty acids in PL. The strongest correlation was between DHA and total fat intake from fish-shellfish $(r \quad 0.38 ; P<0.001)$. The intake of fish-shellfish reflected as the weekly fish intake correlated well (all $r \geq 0.41$ and $P<0.001$ ) with total $n$-3 PUFA, EPA and DHA in plasma PL (Table 5). Correlations between dietary fish-shellfish intake and total $n-3(r 0.24 ; P<0 \cdot 001)$, EPA $(r 0.19 ; P<0.01)$ and DHA $(r 0.29 ; P<0.001)$ in PL were attenuated but remained after adjustment for covariates. However, the relationship between EPA and total fat and PUFA from fish-shellfish disappeared in men. Further, there was a dose-response relationship between the three-level weekly fish intake and total $n-3$ fatty acids in plasma PL in both men and women (ANOVA $P<0 \cdot 001$; data not shown).

The ranking of the fish and shellfish intake in the questionnaire was also fair in comparison with the intake of fishshellfish from the diet record $\left(\kappa_{\mathrm{w}}=0 \cdot 30 ; P<0 \cdot 001\right)$.

Meat and sausage intake was negatively correlated with plasma PL $14: 0(r-0 \cdot 20 ; P=0.02)$ in men and $15: 0$ 
Table 1. Characteristics of study participants

(Number of subjects and percentage, mean values and standard deviations, and medians and interqurtile ranges (IQR))*

\begin{tabular}{|c|c|c|c|c|c|c|c|}
\hline & \multicolumn{2}{|c|}{ All } & \multicolumn{2}{|c|}{ Women } & \multicolumn{2}{|c|}{ Men } & \multirow[b]{2}{*}{$P$ for difference } \\
\hline & Median & IQR & Median & IQR & Median & IQR & \\
\hline \multicolumn{8}{|l|}{ Subjects } \\
\hline$n$ & 279 & & 150 & & 129 & & \\
\hline$\%$ & & & 54 & & 46 & & \\
\hline Age & & & & & & & 0.03 \\
\hline Mean & 50 & & 48 & & 53 & & \\
\hline SD & 17 & & 16 & & 17 & & \\
\hline BMI $\left(\mathrm{kg} / \mathrm{m}^{2}\right)$ & $24 \cdot 8$ & $22 \cdot 6-27.5$ & $24 \cdot 2$ & $22 \cdot 3-27.5$ & $25 \cdot 1$ & $23 \cdot 3-27.5$ & 0.06 \\
\hline \multicolumn{8}{|l|}{ Physical activity level } \\
\hline At work & & & & & & & 0.31 \\
\hline \multicolumn{8}{|l|}{ Sedentary } \\
\hline$n$ & 209 & & 116 & & 93 & & \\
\hline$\%$ & 75 & & 75 & & 72 & & \\
\hline \multicolumn{8}{|l|}{ Heavy } \\
\hline$n$ & 70 & & 34 & & 36 & & \\
\hline$\%$ & 25 & & 25 & & 28 & & \\
\hline At leisure time & & & & & & & 0.99 \\
\hline \multicolumn{8}{|l|}{ Sedentary } \\
\hline$n$ & 132 & & 71 & & 61 & & \\
\hline$\%$ & 47 & & 47 & & 47 & & \\
\hline \multicolumn{8}{|l|}{ Active/regular } \\
\hline$n$ & 147 & & 79 & & 68 & & \\
\hline$\%$ & 53 & & 53 & & 53 & & \\
\hline Current, occasional smokers (\%) & 15 & & 17 & & 13 & & 0.45 \\
\hline Supplement use (fish oil, $n-3$ ) & & & & & & & 0.17 \\
\hline$n$ & 46 & & 29 & & 17 & & \\
\hline$\%$ & 16 & & 19 & & 13 & & \\
\hline Under-reporters (\%) & $13 \cdot 6$ & & 14.0 & & $13 \cdot 2$ & & 0.84 \\
\hline Energy (MJ) & & & & & & & $<0.001$ \\
\hline Mean & $8 \cdot 6$ & & 7.4 & & 9.4 & & \\
\hline SD & 2.4 & & $2 \cdot 0$ & & 2.5 & & \\
\hline \multicolumn{8}{|l|}{ Dietary fat intake } \\
\hline Total fat (g/10 MJ) & 91 & 82-104 & 92 & 82-104 & 90 & $82-103$ & 0.66 \\
\hline SFA $(g / 10 \mathrm{MJ})$ & 35 & $30-40$ & 35 & $30-41$ & 35 & $30-39$ & 0.73 \\
\hline$\%$ Total fatty acids & & & & & & & 0.80 \\
\hline Mean & 38 & & 38 & & 38 & & \\
\hline SD & 5 & & 5 & & 6 & & \\
\hline MUFA (g/10 MJ) & 34 & $30-39$ & 34 & 30-38 & 34 & 30-39 & 0.91 \\
\hline$\%$ Total fatty acids & & & & & & & 0.44 \\
\hline Mean & 37 & & 37 & & 37 & & \\
\hline SD & 3 & & 3 & & 3 & & \\
\hline PUFA (g/10 MJ) & 14 & $12-18$ & 14 & $12-18$ & 14 & $12-17$ & 0.68 \\
\hline$\%$ Total fatty acids & & & & & & & 0.98 \\
\hline Mean & 16 & & 16 & & 16 & & \\
\hline SD & 5 & & 4 & & 5 & & \\
\hline Fat from dairy products (g/10 MJ) & $6 \cdot 7$ & $3 \cdot 2-10 \cdot 3$ & $6 \cdot 9$ & $3 \cdot 7-11 \cdot 3$ & $6 \cdot 0$ & $2 \cdot 7-9.5$ & $0 \cdot 17$ \\
\hline Fat from fish and shellfish ( $\mathrm{g} / 10 \mathrm{MJ})$ & 4.3 & $0 \cdot 3-9.5$ & $4 \cdot 3$ & $0.4-8 \cdot 7$ & $4 \cdot 3$ & $0 \cdot 1-10 \cdot 3$ & 0.75 \\
\hline \multicolumn{8}{|l|}{ Intake of food groups } \\
\hline Fish and shellfish (g/10 MJ) & 44 & $4 \cdot 5-77$ & 43 & $5 \cdot 6-74$ & 47 & $4 \cdot 5-78$ & 0.82 \\
\hline Dairy products† (g/10 MJ) & 319 & $183-431$ & 346 & $216-453$ & 279 & $167-422$ & 0.03 \\
\hline Meat and sausages (g/10 MJ) & 99 & $67-138$ & 97 & $60-131$ & 104 & $72-154$ & 0.07 \\
\hline Spreadsł (g/10 MJ) & 11 & $3 \cdot 9-20$ & 9 & $1 \cdot 3-17$ & 14 & $5 \cdot 5-23$ & $<0.01$ \\
\hline
\end{tabular}

*Mean and standard deviation are reported for normally distributed variables and as median and interquartile range (25th and 75th percentile] for skewed variables. †Dairy products include milk, yoghurt, fermented milk, sour cream, cream and cheese.

†Spreads include different margarines, spread mixtures and butter used on bread or as a side dish (for example on boiled potatoes).

$(r-0 \cdot 18 ; P=0 \cdot 03)$ in women, but not with PL $17: 0$ in either women or men. These correlations were unaffected by adjustments. Intake of spreads did not correlate with any of the plasma PL fatty acids, except for a marginal correlation with $15: 0(r 0.11 ; P=0 \cdot 06)$ (data not shown).

In sensitivity analyses, when under-reporters and supplement users were excluded, respectively, the results were only marginally different (data not shown).

\section{Discussion}

The present study indicates that the web-based 4-d food record, the Riksmaten method, used in the latest Swedish National Dietary Study in adults, appears valid for the purpose of collecting information on dietary fat. Moderate associations between the consumption of longer-chained $n$ - 3 fatty acids as well as fish-shellfish intake and PL levels are reported. Further, the intake of fish and shellfish reported in the 
Table 2. Proportion (\% of total) of fatty acids estimated in plasma phospholipids in all participants $(n 279)$ and in women $(n 150)$ and men $(n 129)$ (Mean values and standard deviations)

\begin{tabular}{|c|c|c|c|c|c|c|}
\hline \multirow[b]{2}{*}{ Fatty acid } & \multicolumn{2}{|c|}{ All } & \multicolumn{2}{|c|}{ Women } & \multicolumn{2}{|c|}{ Men } \\
\hline & Mean & SD & Mean & SD & Mean & SD \\
\hline$\sum$ SFA & $47 \cdot 2$ & 1.6 & $47 \cdot 1$ & 1.6 & $47 \cdot 3$ & 1.7 \\
\hline Myristic acid, $14: 0$ & 0.41 & 0.1 & 0.43 & $0 \cdot 10$ & $0.40^{\star \star}$ & 0.09 \\
\hline Pentadecanoic acid, $15: 0$ & 0.26 & 0.05 & 0.27 & 0.05 & 0.26 & 0.06 \\
\hline Palmitic acid, $16: 0$ & 31.3 & 1.5 & 31.2 & 1.5 & 31.4 & 1.5 \\
\hline Heptadecanoic acid, $17: 0$ & 0.43 & 0.06 & 0.43 & 0.06 & 0.42 & 0.07 \\
\hline Stearic acid, $18: 0$ & 14.8 & 1.2 & $14 \cdot 8$ & 1.2 & 14.8 & 1.2 \\
\hline$\sum$ MUFA & 13.5 & 1.2 & 13.5 & 1.3 & 13.5 & $1 \cdot 2$ \\
\hline Palmitoleic acid, $16: 1 n-7$ & 0.50 & 0.2 & 0.53 & 0.16 & $0.49^{\star \star *}$ & 0.21 \\
\hline Oleic acid, $18: 1$ & $13 \cdot 0$ & $1 \cdot 1$ & $12 \cdot 9$ & $1 \cdot 2$ & $13 \cdot 2$ & 1.4 \\
\hline$\sum$ PUFA & $39 \cdot 3$ & $2 \cdot 0$ & 39.4 & 1.9 & $39 \cdot 2$ & $2 \cdot 1$ \\
\hline$\sum n-6$ & 32.5 & $2 \cdot 2$ & $32 \cdot 6$ & $2 \cdot 1$ & $32 \cdot 3$ & $2 \cdot 2$ \\
\hline Linoleic acid, $18: 2 n-6$ & $21 \cdot 3$ & $2 \cdot 3$ & 21.6 & $2 \cdot 4$ & $20 \cdot 9$ & $2 \cdot 4$ \\
\hline$\gamma$-Linolenic acid, $18: 3 n-6$ & 0.11 & 0.04 & 0.10 & 0.04 & 0.1 & 0.04 \\
\hline Dihomo- $\gamma$-linolenic acid, $20: 3 n-6$ & $2 \cdot 8$ & 0.6 & $2 \cdot 8$ & 0.7 & $2 \cdot 8$ & 0.6 \\
\hline Arachidonic acid, $20: 4 n-6$ & $8 \cdot 3$ & 1.4 & $8 \cdot 2$ & 1.5 & 8.5 & 1.4 \\
\hline$\sum n-3$ & $6 \cdot 8$ & 1.7 & $6 \cdot 7$ & $1 \cdot 8$ & 6.9 & 1.6 \\
\hline$\alpha$-Linolenic acid, $18: 3 n-3$ & 0.28 & 0.1 & 0.29 & 0.1 & $0 \cdot 27^{*}$ & 0.08 \\
\hline EPA, $20: 5 n-3$ & $1 \cdot 7$ & 0.8 & 1.7 & 0.9 & 1.7 & 0.7 \\
\hline Docosapentaenoic acid, $22: 5 n-3$ & 0.93 & 0.2 & 0.91 & 0.18 & $0.96^{\star}$ & 0.17 \\
\hline DHA, $22: 6 n-3$ & 3.9 & 1.0 & 3.9 & 1.1 & 3.9 & 0.98 \\
\hline
\end{tabular}

Mean value was significantly different from that for men: ${ }^{*} P \leq 0.05,{ }^{* *} P \leq 0.01,{ }^{* \star *} P \leq 0.001$.

Table 3. Spearman rank correlations $(r)$ between fatty acid intake estimated in the diet by a 4-d food record and plasma phospholipids, in all study participants ( $n$ 279) and by sex $\dagger$

\begin{tabular}{|c|c|c|c|}
\hline Fatty acid & All & Women & Men \\
\hline Total SFA & -0.07 & -0.08 & -0.07 \\
\hline Myristic acid, $14: 0$ & $0.16^{* *}$ & 0.10 & $0.21^{*}$ \\
\hline Palmitic acid, $16: 0$ & -0.02 & -0.07 & 0.05 \\
\hline Stearic acid, $18: 0$ & -0.03 & 0.04 & -0.13 \\
\hline Total MUFA & -0.11 & -0.04 & $-0.21^{*}$ \\
\hline Palmitoleic acid, $16: 1$ & -0.03 & 0.10 & -0.10 \\
\hline Oleic acid, $18: 1 n-7$ & -0.10 & -0.04 & -0.16 \\
\hline Total PUFA & $0.12^{*}$ & $0 \cdot 17^{*}$ & 0.07 \\
\hline Total $n-6$ fatty acids & 0.10 & 0.08 & 0.13 \\
\hline Linoleic acid, $18: 2 n-6$ & 0.07 & 0.15 & -0.03 \\
\hline Arachidonic acid, $20: 4 n-6$ & 0.07 & $0 \cdot 10$ & 0.02 \\
\hline Total $n-3$ fatty acids & $0.31^{\star \star \star}$ & $0.33^{\star \star \star}$ & $0.28^{\star \star \star}$ \\
\hline$\alpha$-Linolenic acid, $18: 3 n-3$ & 0.07 & 0.09 & 0.05 \\
\hline EPA, $20: 5 n-3$ & $0.34^{\star \star \star}$ & $0.36^{\star \star \star}$ & $0.28^{\star \star \star}$ \\
\hline Docosapentaenoic acid, $22: 5 n-3$ & 0.05 & 0.06 & 0.009 \\
\hline DHA, $22: 6 n-3$ & $0.42^{\star * *}$ & $0.44^{\star * *}$ & $0.39^{* * *}$ \\
\hline
\end{tabular}

${ }^{*} P \leq 0.05,{ }^{* *} P \leq 0.01,{ }^{* * *} P \leq 0.001$.

†Pentadecanoic acid (15:0), heptadecanoic acid (17:0), $\gamma$-linolenic acid (18:3n-6) and dihomo- $\gamma$-linolenic acid $(20: 3 n-6)$ could not be determined in the diet and were thus not correlated in this analysis.

questionnaire correlated with the intake of fish and shellfish from the diet record as well as with plasma EPA and DHA. We also found a dose-response relationship between fish consumption and levels of $n-3$ fatty acids in the blood, consistent with previous studies ${ }^{(27,28)}$. The present study also supports that myristic acid $(14: 0)$ is a valid marker for dairy food intake, but surprisingly we did not find a relationship between PL 14 : 0 and dairy fat. This may be because myristic acid only makes up a small proportion of dairy fat ${ }^{(29)}$ and that 14 : 0 is present in other dietary sources such as coconut oil. We did, however, find a significant positive correlation between PL 14 : 0 and dietary 14 : 0. Pentadecaenoic acid (15:0) was correlated with both intake of dairy products and amount of fat from dairy products while heptadecaenoic acid (17:0) was related to the amount of fat from dairy products, mainly in women.

The proportions of plasma PL fatty acids reported in this national dietary study were in the same range as reported in other Swedish studies and analysed in the same laboratory ${ }^{(30-32)}$. However, the proportion of plasma DHA in the present study was lower compared with that reported in previous Swedish studies ${ }^{(30-32)}$. Participants who had not eaten fish during the registration period had lower proportions of plasma EPA and DHA, which is consistent with previous observations ${ }^{(27)}$, and possibly explains the detected lower proportions in the study population. The proportions of EPA and DHA were positively related to age and this is in agreement with a higher consumption of fish-shellfish at higher ages ${ }^{(7)}$. Those who consumed fish-oil and $n$-3 supplements had higher levels of EPA and DHA, but reported likewise a higher intake of fish-shellfish. Less than $20 \%$ of the study population used supplements and exclusion of these individuals from the analysis had only marginal effects on the results.

There was no correlation between dietary and plasma PL LA when all study participants were examined, but there was a marginal correlation in women $(r 0.15 ; P=0.06)$. This is in contrast to previous studies ${ }^{(25,26)}$, but not in all ${ }^{(33)}$. It is possible that the diet record did not adequately measure LA intake, or that the intake range was too narrow to detect a correlation. A preferential misreporting of foods high in LA is possible. LA was the largest individual PUFA in the diet and is a precursor for longer and more unsaturated $n-6$ fatty acids like arachidonic acid (20:4n-6), a precursor for eicosanoids $^{(15)}$. This endogenous conversion as well as oxidation of LA may have affected the correlation between diet and biomarker. We could not retrieve information about the amount 
Table 4. Crude and adjusted + Spearman rank correlations ( $r$ ) between specific plasma phospholipid fatty acids and dairy products in all study participants $(n$ 279) and by sex

\begin{tabular}{|c|c|c|c|c|c|c|c|c|c|c|}
\hline \multirow[b]{2}{*}{ Fatty acid } & & \multicolumn{3}{|c|}{ Dairy products $\ddagger(g / d)$} & \multicolumn{3}{|c|}{ Total fat from dairy products $\ddagger(\mathrm{g} / \mathrm{d})$} & \multicolumn{3}{|c|}{ SFA from dairy products $\ddagger(g / d)$} \\
\hline & & All & Women & Men & All & Women & Men & All & Women & Men \\
\hline \multirow[t]{2}{*}{$14: 0$} & Crude & $0.15^{\star *}$ & 0.08 & $0.19^{*}$ & 0.07 & -0.006 & 0.12 & 0.008 & -0.007 & 0.13 \\
\hline & Adjusted & 0.12 & 0.10 & 0.15 & 0.02 & 0.03 & 0.03 & 0.02 & 0.03 & 0.04 \\
\hline \multirow[t]{2}{*}{$15: 0$} & Crude & $0.25^{\star \star *}$ & 0.10 & $0.36^{\star \star *}$ & $0.32^{\star * *}$ & $0.20^{\star *}$ & $0.43^{\star \star *}$ & $0.31^{* \star *}$ & $0 \cdot 18^{*}$ & $0.44^{\star * \star}$ \\
\hline & Adjusted & $0.22^{\star \star *}$ & $0.24^{*}$ & $0.19^{\star}$ & 0.09 & 0.07 & $0 \cdot 16$ & 0.10 & 0.06 & $0.18^{\star}$ \\
\hline \multirow[t]{2}{*}{$17: 0$} & Crude & 0.08 & 0.10 & 0.05 & $0 \cdot 16^{\star *}$ & $0.17^{*}$ & 0.15 & $0 \cdot 16^{\star *}$ & $0 \cdot 16^{*}$ & 0.15 \\
\hline & Adjusted & 0.11 & $0.21^{\star *}$ & -0.06 & 0.10 & $0.20^{*}$ & -0.04 & 0.11 & $0.19^{*}$ & -0.02 \\
\hline
\end{tabular}

${ }^{\star} P \leq 0.05,{ }^{* *} P \leq 0.01,{ }^{* * *} P \leq 0.001$

†Adjusted for BMI, smoking habits, physical activity level, alcohol intake and age.

$\ddagger$ Residual adjusted.

Table 5. Crude Spearman rank correlations ( $r$ ) between specific plasma phospholipid fatty acids and dairy products and meat and in all study participants $(n$ 279) and by sex

\begin{tabular}{|c|c|c|c|c|c|c|c|c|c|c|c|c|}
\hline \multirow[b]{2}{*}{ Fatty acid } & \multicolumn{3}{|c|}{ Fish and shellfish $\dagger(\mathrm{g} / \mathrm{d})$} & \multicolumn{3}{|c|}{$\begin{array}{l}\text { Total fat from fish and } \\
\text { shellfish } \dagger(\mathrm{g} / \mathrm{d})\end{array}$} & \multicolumn{3}{|c|}{$\begin{array}{l}\text { PUFA from fish and shellfish } \dagger \\
\qquad(\mathrm{g} / \mathrm{d})\end{array}$} & \multicolumn{3}{|c|}{ Weekly fish intake $\neq$} \\
\hline & All & Women & Men & All & Women & Men & All & Women & Men & All & Women & Men \\
\hline Total $n-3$ & $0.35^{\star \star *}$ & $0.36^{\star \star \star}$ & $0.35^{\star \star *}$ & $0.34^{\star * *}$ & $0.39^{\star \star \star}$ & $0.29^{\star \star \star}$ & $0.33^{\star \star \star}$ & $0.38^{\star \star \star}$ & $0.29^{* * *}$ & $0.45^{\star \star *}$ & $0.50^{\star \star *}$ & $0.38^{\star \star *}$ \\
\hline $20: 5 n-3$ & $0.31^{\star \star \star}$ & $0.33^{\star * *}$ & $0.29^{\star \star \star}$ & $0.26^{\star \star \star}$ & $0.34^{\star \star *}$ & $0.17^{\star}$ & $0.25^{* * *}$ & $0.32^{\star \star *}$ & $0.17^{\star}$ & $0.41^{* * *}$ & $0.47^{* * *}$ & $0.30^{* * *}$ \\
\hline $22: 6 n-3$ & $0.36^{* * *}$ & $0.36^{\star \star *}$ & $0.34^{* * *}$ & $0.38^{* \star *}$ & $0.42^{* * *}$ & $0.34^{\star \star *}$ & $0.37^{* * *}$ & $0.42^{\star * *}$ & $0.33^{* * *}$ & $0.44^{\star * *}$ & $0.47^{* * *}$ & $0.40^{* \star *}$ \\
\hline
\end{tabular}

${ }^{*} P \leq 0.05,{ }^{* \star *} P<0.001$.

†Residual adjusted.

‡From questionnaire.

and type of cooking fat used in dishes and for baking from the diet record, due to technical reasons. It is possible that this may partly explain the lack of correlation between PL and dietary LA. Also, the use of standard fat in recipes in the diet record may have influenced the result. These reasons may explain the lack of correlation between dietary $\alpha$-linolenic acid (18 : $3 n-3)$ and plasma levels in the present study as well.

That tissue SFA is weakly correlated with diet is partly related to the rapid endogenous conversion to MUFA from SFA by desaturases ${ }^{(21)}$. Further, MUFA was negatively correlated with dietary intake partly because of the endogenous production of MUFA. Biomarker MUFA correlated with SFA and this has been reported previously ${ }^{(26)}$. This relates to the fact that the intake of oleic acid in Sweden, as well as in for example the USA, is mainly derived from dairy products and meat which also are main sources of SFA.

In the present study, plasma PL $15: 0$ and $17: 0$ were correlated with the intake of dairy products and partly to dairy fat as previously suggested ${ }^{(24,34,35)}$, although the correlations differed between the sexes. Different species of fish contain $15: 0$ and $17: 0$ of varying content ${ }^{(36)}$ and a previous study (European Prospective Investigation into Cancer and Nutrition; EPIC) reported positive associations between tissue $15: 0$ and $17: 0$ and fish intake on an ecological level ${ }^{(37)}$. However, we found no correlation between milk fat biomarkers and fish intake in the present cross-sectional study.

The intake of meat and sausages was not correlated with tissue $15: 0$ and $17: 0$, probably because the intake of pork and pork-containing sausages exceeds the intake of ruminant meat in the survey.
The magnitude of the correlations between individual fatty acids in PL and diet was in the present study as what can be expected with only one diet measurement and in the same range as previously reported ${ }^{(25,26,38)}$. Correlations between fatty acids measured in plasma PL and diet differed in men and women, despite similar proportions in plasma. It is possible that these results are due to chance or it is possible that fatty acids from different dietary sources affect metabolism differently. Further, it is possible that accuracy of reporting is different among men and women, which was partly confirmed in the interaction analysis. It is further known that fat metabolism is different in men and women ${ }^{(39)}$. Non-dietary factors said to affect plasma fatty acid composition ${ }^{(21)}$, such as adiposity and age, differed between the sexes and may have influenced the results, but this was accounted for in the analyses.

The strengths of the present study include the random population sample that included women and men from all parts of Sweden, the careful building of the food list in the web-application and that data was quality controlled before analysis. Further, the web-based dietary record was selfassisted, which minimised external coding errors. This could be a limitation since self-reporting could be considered as if there were 279 individual reporters/coders. However, this introduces a random error whereas using interviewers for coding could introduce a systematic error. On the other hand, interviewers might be able to retrieve more detailed information on foods compared with self-reporting. Another limitation is that the participation rate was low; this could have introduced bias. Participants had a higher degree of 
educational attainment than those who did not participate and few subjects with immigrant background participated ${ }^{(7)}$. This limitation relates, however, more to the dietary survey rather than to the present relative validation. Under-reporting may influence the results in any diet study, but when underreporters were excluded from our analyses the interpretation of the results remained unchanged. Another limitation is that we did not measure fatty acids in any other fraction, for example the cholesteryl ester fraction. It is possible that our 4-d diet record did not reflect the fat intake representing the same period as the fatty acids in the PL fraction.

Dietary data (food consumption data) collected in a national dietary survey are used for several purposes: to monitor nutrient and food intakes in the population as well as to carry out food-based risk-benefit assessments and policy making within the European Union. It is therefore important that the data collected meet the requirements set out both on a national level as well as by the European Food Safety Agency ${ }^{(40)}$ and are as accurate as possible. In conclusion, the Riksmaten method appears valid for the purpose of collecting data on dietary fat composition derived from several core food groups, at least in a healthy adult population.

\section{Acknowledgements}

The authors thank Monika Pearson for all the work with the national dietary study and Ingalill Gadhasson for her valuable contribution taking care of the biological samples. We thank the staff at the Occupational and Environmental Medicine Centres for the collection of samples, as well as all volunteers for participating in the study.

Financial support from the Swedish Environmental Protection Agency made the collection of biological samples in the Swedish National Dietary Survey, Riksmaten adults 2010-11 possible.

There are no conflicts of interest.

\section{References}

1. Willett WC \& Stampfer MJ (2013) Current evidence on healthy eating. Annu Rev Public Health 34, 77-95.

2. Calder PC (2013) Long chain fatty acids and gene expression in inflammation and immunity. Curr Opin Clin Nutr Metab Care 16, 425-433.

3. Nordic Council of Ministers (2013) Nordic Nutrition Recommendations 2012. Part 1. Summary, Principles and Use, 5th ed. Copenhagen: Nordic Council of Ministers.

4. Kromhout D, Geleijnse JM, Menotti A, et al. (2011) The confusion about dietary fatty acids recommendations for CHD prevention. $\mathrm{Br}$ J Nutr 106, 627-632.

5. Becker W, Darnerud PO \& Petersson-Grawé K (2007) Risks and Benefits of Fish Consumption. National Food Agency Report, no. 12. Uppsala: National Food Agency.

6. Mozaffarian D \& Wu JH (2011) Omega-3 fatty acids and cardiovascular disease: effects on risk factors, molecular pathways, and clinical events. J Am Coll Cardiol 58, 2047-2067.

7. Amcoff E, Edberg A, Barbieri HE, et al. (2012) Riksmaten-vuxna 2010-11. Livsmedels- och näringsintag bland vuxna i Sverige (Riksmaten-adults 2010-11. Food and Nutrient Intake Among Adults in Sweden). Uppsala: Livsmedelsverket. http://www.livsmedelsverket. se/globalassets/matvanor-halsa-miljo/kostrad-matvanor/matvaneunder sokningar/riksmaten_2010_20111.pdf?id=3588 (accessed March 2015).

8. Kipnis V, Subar AF, Midthune D, et al. (2003) Structure of dietary measurement error: results of the OPEN biomarker study. Am J Epidemiol 158, 14-21; discussion 22-26.

9. Subar AF, Kipnis V, Troiano RP, et al. (2003) Using intake biomarkers to evaluate the extent of dietary misreporting in a large sample of adults: the OPEN study. Am J Epidemiol 158, 1-13.

10. Macdiarmid J \& Blundell J (1998) Assessing dietary intake: who, what and why of under-reporting. Nutr Res Rev 11, 231-253.

11. Livingstone MB \& Black AE (2003) Markers of the validity of reported energy intake. J Nutr 133, Suppl. 3, 895S-920S.

12. Lissner L, Heitmann BL \& Lindroos AK (1998) Measuring intake in free-living human subjects: a question of bias. Proc Nutr Soc 57, 333-339.

13. Goris AH, Westerterp-Plantenga MS \& Westerterp KR (2000) Undereating and underrecording of habitual food intake in obese men: selective underreporting of fat intake. Am J Clin Nutr 71, 130-134.

14. Willett WC (editor) (1998) Nutritional Epidemiology, 2nd ed. New York: Oxford University Press.

15. Arab L (2003) Biomarkers of fat and fatty acid intake. J Nutr 133, Suppl. 3, 925S-932S.

16. Hodson L, Skeaff CM \& Fielding BA (2008) Fatty acid composition of adipose tissue and blood in humans and its use as a biomarker of dietary intake. Prog Lipid Res 47, 348-380.

17. Glatz JF, Soffers AE \& Katan MB (1989) Fatty acid composition of serum cholesteryl esters and erythrocyte membranes as indicators of linoleic acid intake in man. Am J Clin Nutr 49, 269-276.

18. Katan MB, Deslypere JP, van Birgelen AP, et al. (1997) Kinetics of the incorporation of dietary fatty acids into serum cholesteryl esters, erythrocyte membranes, and adipose tissue: an 18-month controlled study. J Lipid Res 38, 2012-2022.

19. Zock PL, Mensink RP, Harryvan J, et al. (1997) Fatty acids in serum cholesteryl esters as quantitative biomarkers of dietary intake in humans. Am J Epidemiol 145, 1114-1122.

20. Baylin A, Kabagambe EK, Siles X, et al. (2002) Adipose tissue biomarkers of fatty acid intake. Am J Clin Nutr 76, 750-757.

21. Baylin A \& Campos H (2006) The use of fatty acid biomarkers to reflect dietary intake. Curr Opin Lipidol 17, 22-27.

22. Black AE (2000) Critical evaluation of energy intake using the Goldberg cut-off for energy intake:basal metabolic rate. A practical guide to its calculation, use and limitations. Int J Obes Relat Metab Disord 24, 1119-1130.

23. Willett WC, Howe GR \& Kushi LH (1997) Adjustment for total energy intake in epidemiologic studies. Am J Clin Nutr 65, 1220S-1228S; discussion 1229S-1231S

24. Smedman AE, Gustafsson IB, Berglund LG, et al. (1999) Pentadecanoic acid in serum as a marker for intake of milk fat: relations between intake of milk fat and metabolic risk factors. $A m \mathrm{~J}$ Clin Nutr 69, 22-29.

25. Hodge AM, Simpson JA, Gibson RA, et al. (2007) Plasma phospholipid fatty acid composition as a biomarker of habitual dietary fat intake in an ethnically diverse cohort. Nutr Metab Cardiovasc Dis 17, 415-426.

26. Ma J, Folsom AR, Shahar E, et al. (1995) Plasma fatty acid composition as an indicator of habitual dietary fat intake in middle-aged adults. The Atherosclerosis Risk in Communities (ARIC) Study Investigators. Am J Clin Nutr 62, 564-571.

27. Masson S, Marchioli R, Mozaffarian D, et al. (2013) Plasma n-3 polyunsaturated fatty acids in chronic heart failure in the GISSI-Heart Failure Trial: relation with fish intake, circulating biomarkers, and mortality. Am Heart J 165, 208-215.e4.

28. Raatz SK, Rosenberger TA, Johnson LK, et al. (2013) Dose-dependent consumption of farmed Atlantic salmon (Salmo salar) increases plasma phospholipid $n-3$ fatty acids differentially. J Acad Nutr Diet 113, 282-287.

29. Lindmark-Månsson H, Fondén R \& Pettersson H-E (2003) Composition of Swedish dairy milk. Int Dairy J 13, 409-425. 
30. Sodergren E, Gustafsson IB, Basu S, et al. (2001) A diet containing rapeseed oil-based fats does not increase lipid peroxidation in humans when compared to a diet rich in saturated fatty acids. Eur J Clin Nutr 55, 922-931.

31. Warensjo E, Riserus U, Gustafsson IB, et al. (2008) Effects of saturated and unsaturated fatty acids on estimated desaturase activities during a controlled dietary intervention. Nutr Metab Cardiovasc Dis 18, 683-690.

32. Wennberg M, Bergdahl IA, Hallmans G, et al. (2011) Fish consumption and myocardial infarction: a second prospective biomarker study from northern Sweden. Am J Clin Nutr 93, 27-36.

33. Swierk M, Williams PG, Wilcox J, et al. (2011) Validation of an Australian electronic food frequency questionnaire to measure polyunsaturated fatty acid intake. Nutrition 27, 641-646.

34. Wolk A, Furuheim M \& Vessby B (2001) Fatty acid composition of adipose tissue and serum lipids are valid biological markers of dairy fat intake in men. J Nutr 131, 828-833.
35. Warensjo E, Nolan D \& Tapsell L (2010) Dairy food consumption and obesity-related chronic disease. Adv Food Nutr Res 59, 1-41.

36. Ozogul Y, Ozogul F, Cicek E, et al. (2009) Fat content and fatty acid compositions of 34 marine water fish species from the Mediterranean Sea. Int J Food Sci Nutr 60, 464-475.

37. Saadatian-Elahi M, Slimani N, Chajes V, et al. (2009) Plasma phospholipid fatty acid profiles and their association with food intakes: results from a cross-sectional study within the European Prospective Investigation into Cancer and Nutrition. Am J Clin Nutr 89, 331-346.

38. Serra-Majem L, Nissensohn M, Overby NC, et al. (2012) Dietary methods and biomarkers of omega 3 fatty acids: a systematic review. Br J Nutr 107, Suppl. 2, S64-S76.

39. Blaak E (2001) Gender differences in fat metabolism. Curr Opin Clin Nutr Metab Care 4, 499-502.

40. European Food Safety Authority (2009) General principles for the collection of national food consumption data in the view of a pan-European dietary survey. EFS A J 7, 1435. 